\title{
Localization of Phosphorylated Proteins Involved in the Regulation of Corneal Cell Proliferation in Response to Wounding
}

\author{
Jenny Chen, Audrey E.K. Hutcheon, James D. Zieske \\ Schepens Eye Research Institute, 20 Staniford St., Boston, MA 02114
}

Previous experiments have suggested that the activation of epidermal growth factor receptors (EGFR) plays a key role in the regulation of corneal epithelial wound healing, but it has not been shown which cells are activated and how long they stay activated. This regulation entails controlling two important aspects of wound healing - the proliferation and migration of cells into the wound area - and it is thought that EGF plays an important role in signaling both actions [1]. In order to research these questions, rat corneal debridement (epithelial removal) wound tissue was stained with antibodies against both total EGFR and phosphorylated EGFR (activated or pEGFR). The localization of total and activated EGFR during the immediate stages of wound healing was analyzed via immunofluorescence microscopy with a TCS SP2 confocal microscope (Leica; Heidelberg, Germany). The time points used were 0, 5, 15, 30, 60, 90, and 120 minutes after a corneal debridement wound. To determine whether there exists a different temporal pattern of activation at the edge versus the cells adjacent to the wound, we defined four regions of the cornea, examined their respective rates of activation, and qualitatively compared the different areas.

In earlier studies, it was found that given a high EGF concentration stimulus, EGFRs appear to complex and aggregate within minutes and become internalized by $30 \mathrm{~min}$ [1]. Our experiments showed that in the leading edge, binding of pEGFR antibody increased significantly within minutes, peaked around $60 \mathrm{~min}$, but was noticeably diminished by $120 \mathrm{~min}$. In cells further away from the wound area, the increase in activated EGFR was minimal, if any, throughout these early time points (Fig. 1). Since we know that cells at the leading edge will eventually migrate to cover the open wound area whereas distal cells proliferate, but do not migrate [2], these data suggest that EGF may be involved in the stimulation of migration, but play a smaller role than previously thought in the initiation of proliferation.

EGFRs exist on the membranes of all corneal epithelial cells (Fig. 2), but during the initial stages following a debridement wound, only the EGFR of basal epithelial cells become activated (Fig. 1). One explanation is that the Heparin-binding-EGF prevalent in tears binds with strong affinity to heparan sulfate proteoglycans in the basement membrane, and consequently is a specific activator of EGFRs on the surface of adjacent basal cells. Secondly, the physiology of the wounded cornea may allow for tears to penetrate more easily via the wound opening along the basement membrane underneath the epithelial layer, perhaps due to the dissociation of hemidesmosomes [3], than directly through the mucoid layer covering the epithelial layer. Therefore it is in this basement membrane area of the cornea that we see the most EGFR activation.

Very little activation of EGFR in cells distal to the wound correlates with non-migratory cells. What remains to be explored is whether EGF, in addition to its involvement in cell migration, is an important cell signaling mechanism for the proliferation of peripheral cells during wound repair. Longer time points should be tested to answer these questions of EGF's role in the regulation of cell proliferation during corneal wound healing. 
References

[1] J.D. Zieske et al., Investigative Ophthalmology \& Visual Science Vol. 41 No. 6 (2000) 1346.

[2] E-H Chung et al., Investigative Ophthalmology \& Visual Science Vol. 40 No. 9 (1999) 1952.

[3] I.K. Gipson et al., Experimental Cell Research Vol. 207 No. 1 (1993) 86.
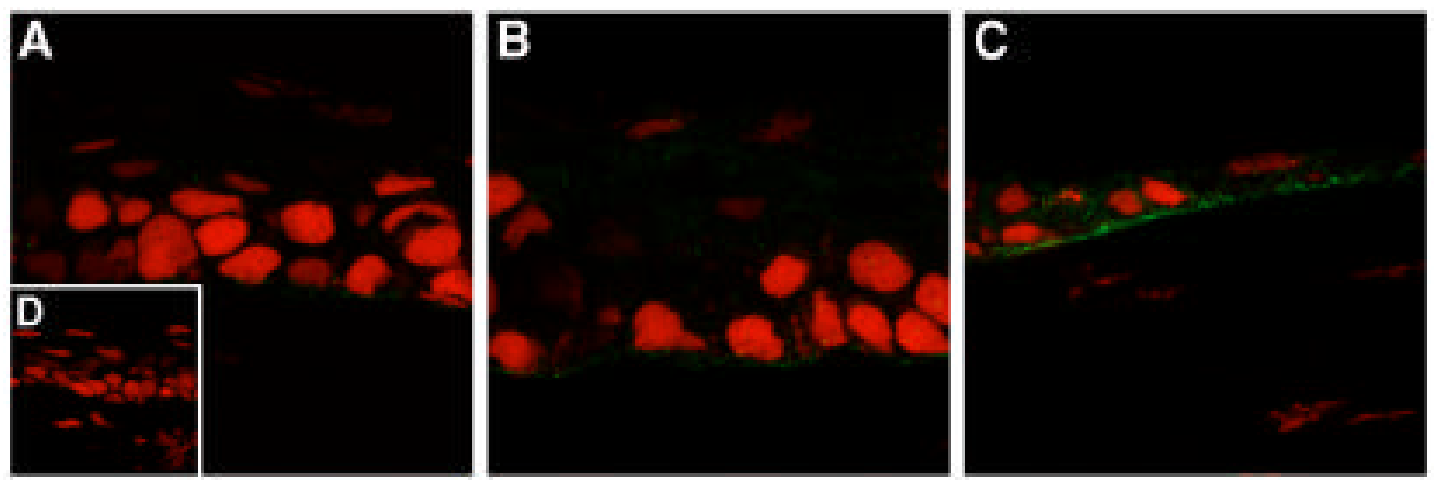

FIG. 1 Immunolocalization of phosphorylated epidermal growth factor receptor (green) in rat corneal epithelium at the periphery (A), near leading edge (B), and leading edge (C) 60 minutes post debridement wound. Tissue is counterstained with propidium iodide (red) to mark cell nucei. The periphery shows no significant increase compared to unwounded control (D).

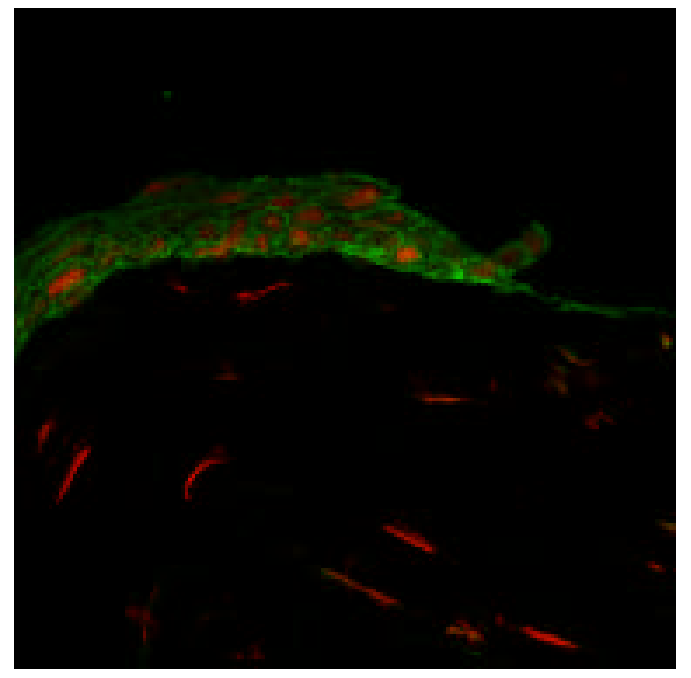

FIG. 2 Immunolocalization of epidermal growth factor receptor (green) in rat corneal epithelium at the wound edge, 5 minutes after a scrape wound. Tissue is counterstained with propidium iodide (red) to mark cell nucei 